\title{
COLLAPSED ISOPARAMETRIC ELEMENT AS A SINGULAR ELEMENT FOR A CRACK NORMAL TO THE BI-MATERIAL INTERFACE
}

\author{
YONG-LI WU \\ Institute of Mechanics, Chinese Academy of Sciences, Beijing 100080 , \\ People's Republic of China
}

(Received 27 April 1992)

\begin{abstract}
It is shown that the variable power singularity of the strain field at the crack tip can be obtained by the simple technique of collapsing quadrilateral isoparametric elements into triangular elements around the crack tip and adequately shifting the side-nodes adjacent to this crack tip. The collapsed isoparametric elements have the desired singularity at crack tip along any ray. The strain expressions for a single element have been derived and in addition to the desired power singularity, additional singularities are revealed. Numerical examples have shown that triangular elements formed by collapsing one side lead to excellent results.
\end{abstract}

\section{INTRODUCTION}

Zak and Williams [1] and Cook and Erdogan [2] have shown that when a crack is normal to a bi-material interface the strain singularity is $r^{\lambda-1}$ with $\lambda(0<\lambda<1)$ the lowest root of the characteristic equation depending on the elastic constants of the two materials. The accurate numerical solution of problems involving a crack tip singularity $r^{i-1}$ requires an accurate modeling of the singularity. Various methods for generating variable power singular elements have been developed [3-7]. Recently El Abdi and Valentin [8] have shown that isoparametric elements with a suitable choice of position of the side-nodes adjacent to the crack tip can introduce any required singularity $(\lambda-1)$. The advantages of the isoparametric element are that the displacement compatibility is satisfied throughout the region and there is no need of special crack tip elements. However, the strains of the quadrilateral isoparametric elements have the singularity only on two sides of the elements. Based on the investigation of El Abdi and Valentin, this paper shows that the variable power singularity of strain field at the crack tip along any ray can be obtained by a simple method of collapsing the quadrilateral element into a triangular element and adequately shifting the side-nodes adjacent to this crack tip. The detailed strain expressions of the singular element have been given and in addition to desired power singularity $r^{\lambda-1}$, additional singularity $r^{2 \lambda-1}$ for the quadratic element and singularities $r^{2 i-1}, r^{3 i-1}$ for the cubic element are revealed. Numerical examples are given to demonstrate the applicability of the collapsed isoparametric elements for perpendicular interface crack.

\section{QUADRATIC ISOPARAMETRIC ELEMENT}

Consider a quadratic isoparametric element

$$
\begin{aligned}
& x=\sum_{i=1}^{8} N_{i} x_{i}, \quad y=\sum_{i=1}^{8} N_{i} y_{i} \\
& u=\sum_{i=1}^{8} N_{i} u_{i}, \quad v=\sum_{i=1}^{8} N_{i} v_{i},
\end{aligned}
$$

where $N_{i}$ are shape functions and are given by [9]. Corner nodes

$$
N_{1}=-\frac{1}{4}(1-\xi)(1-\eta)(1+\xi+\eta), \text { etc. }
$$

Mid-side nodes

$$
N_{5}=-\frac{1}{2}\left(1-\xi^{2}\right)(1-\eta), \text { etc. }
$$

Without loss of generality consider the collapsed triangular element, together with the mapped unit element in the transformed plane, shown in Fig. 1. We have

$$
x_{1}=x_{4}=x_{8}=0, \quad y_{1}=y_{4}=y_{8}=0 .
$$

Assume

$$
x_{5}=\alpha x_{2}, \quad x_{7}=\alpha x_{3}
$$

Substituting (3), (4), (5) into (1) and collecting terms we get

$$
x=\frac{1}{2}\left[x_{2}(1-\eta)+x_{3}(1+\eta)\right]\left[\alpha+\frac{1}{2} \xi+\left(\frac{1}{2}-\alpha\right) \xi^{2}\right] .
$$




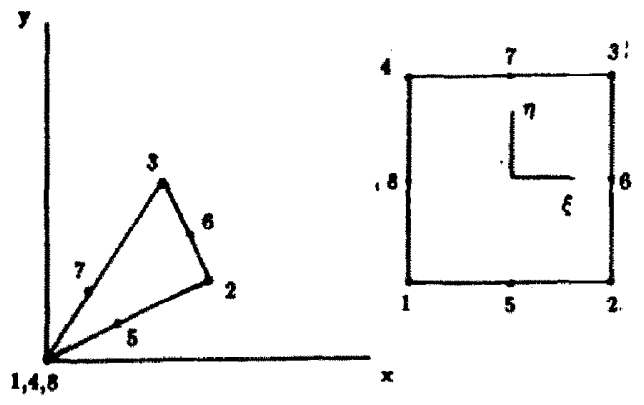

Fig. 1. Collapsed quadratic isoparametric element and mapped unit element.

The least-squares method can be used to determine $\alpha$ values for various $\lambda$ values by satisfying condition [8]

$$
\alpha+\frac{1}{2} \xi+\left(\frac{1}{2}-\alpha\right) \xi^{2}=\left(\frac{1+\xi}{2}\right)^{1 / \alpha}, \text { for }-1 \leqslant \xi \leqslant 1 .
$$

Equation (6) can be written

$$
x=\frac{1}{2}\left[x_{2}(1-\eta)+x_{3}(1+\eta)\right]\left(\frac{1+\xi}{2}\right)^{1 / 2} .
$$

Similarly we have

$$
y=\frac{1}{2}\left[y_{2}(1-\eta)+y_{3}(1+\eta)\right]\left(\frac{1+\xi}{2}\right)^{1 / 2} .
$$

Yamada et al. [10] have given the same relation between Cartesian coordinates $x, y$ and the parametric one $\xi, \eta$ by introducing singularity mapping. From (8) and (9) we get

$$
\eta=\frac{\left(\tan \theta_{1}+k \tan \theta_{2}\right)-(k+1) \tan \theta}{(k-1) \tan \theta+\left(\tan \theta_{1}-\tan \theta_{2}\right)}
$$

where

$\tan \theta=\frac{y}{x}, \quad \tan \theta_{1}=\frac{y_{2}}{x_{2}}, \quad \tan \theta_{2}=\frac{y_{3}}{x_{3}}, \quad k=\frac{x_{3}}{x_{2}}$.

Substituting eqn (10) into eqn (8) we get

$$
\xi=-1+A r^{\grave{\lambda}},
$$

where

$$
A(\theta)=2\left[\frac{(1-k) \sin \theta+\left(k \tan \theta_{2}-\tan \theta_{1}\right) \cos \theta}{x_{3}\left(\tan \theta_{2}-\tan \theta_{1}\right)}\right]^{i} .
$$

From eqns (10) and (12)

$$
\begin{aligned}
& \frac{\partial \xi}{\partial x}=\frac{\partial \xi}{\partial r} \frac{\partial r}{\partial x}+\frac{\partial \xi}{\partial \theta} \frac{\partial \theta}{\partial x}=f_{1}(\theta) r^{i-1} \\
& \frac{\partial \xi}{\partial y}=\frac{\partial \xi}{\partial r} \frac{\partial r}{\partial y}+\frac{\partial \xi}{\partial \theta} \frac{\partial \theta}{\partial y}=f_{2}(\theta) r^{i-1} \\
& \frac{\partial \eta}{\partial y}=\frac{\partial \eta}{\partial y} \frac{\partial y}{\partial x}+\frac{\partial \eta}{\partial \theta} \frac{\partial \theta}{\partial y}=f_{3}(\theta) r^{\lambda_{1}} \\
& \frac{\partial \eta}{\partial y}=\frac{\partial \eta}{\partial r} \frac{\partial r}{\partial y}+\frac{\partial \eta}{\partial \theta} \frac{\partial \theta}{\partial y}=f_{4}(\theta) r^{i-1}
\end{aligned}
$$

where

$$
\begin{aligned}
& f_{1}(\theta)=\frac{A\left(k \tan \theta_{2}-\tan \theta_{1}\right) \lambda}{(1-k) \sin \theta+\left(k \tan \theta_{2}-\tan \theta_{1}\right) \cos \theta} \\
& f_{2}(\theta)=\frac{(k-1) \cos 2 \theta+\left(k \tan \theta_{2}-\tan \theta_{1}\right) \sin 2 \theta}{(1-k) \sin \theta+\left(k \tan \theta_{2}-\tan \theta_{1}\right) \cos \theta} A \lambda
\end{aligned}
$$

$f_{3}(\theta)=\frac{-2 k\left(\tan \theta_{2}-\tan \theta_{1}\right) \sin \theta}{\left[(k-1) \tan \theta+\left(\tan \theta_{1}-k \tan \theta_{2}\right)\right]^{2} \cos ^{2} \theta}$

$f_{4}(\theta)=\frac{2 k\left(\tan \theta_{2}-\tan \theta_{1}\right)}{\left[(k-1) \tan \theta+\left(\tan \theta_{1}-k \tan \theta_{2}\right)\right]^{2} \cos \theta}$.

Next we consider the strain of the collapsed triangular element. The strains are given by the chain rule

$$
\begin{aligned}
& \epsilon_{x}=\frac{\partial u}{\partial x}=\frac{\partial u}{\partial \xi} \frac{\partial \xi}{\partial x}+\frac{\partial u}{\partial \eta} \frac{\partial \eta}{\partial x} \\
& \epsilon_{y}=\frac{\partial v}{\partial y}=\frac{\partial v}{\partial \xi} \frac{\partial \xi}{\partial y}+\frac{\partial v}{\partial \eta} \frac{\partial \eta}{\partial y} \\
& \gamma_{x y}=\frac{\partial u}{\partial y}+\frac{\partial v}{\partial x}=\frac{\partial u}{\partial \xi} \frac{\partial \xi}{\partial y}+\frac{\partial u}{\partial \eta} \frac{\partial \eta}{\partial y}+\frac{\partial v}{\partial \xi} \frac{\partial \xi}{\partial x}+\frac{\partial v}{\partial \eta} \frac{\partial \eta}{\partial x}
\end{aligned}
$$

and $\partial u / \partial \xi, \partial u / \partial \eta, \partial v / \partial \xi, \partial v / \partial \eta$, involved are obtainable by differentiating eqn (2) and using eqn (10), (12)

$$
\frac{\partial u}{\partial \xi}=B_{1} r^{\lambda}+B_{2}, \quad \frac{\partial u}{\partial \eta}=B_{3} r^{2 \lambda}+B_{4} r^{\lambda}+B_{5}
$$

$$
\frac{\partial v}{\partial \xi}=C_{1} r^{i}+C_{2}, \frac{\partial v}{\partial \eta}=C_{3} r^{2 i}+C_{4} r^{i}+C_{5}
$$

with

$$
\begin{aligned}
B_{1}= & \frac{A}{2}\left[\left(u_{1}+u_{2}+u_{3}+u_{4}-2 u_{5}-2 u_{7}\right)\right. \\
& \left.+\eta\left(u_{3}+u_{4}-2 u_{5}-u_{1}-u_{2}-2 u_{7}\right)\right]
\end{aligned}
$$




$$
\begin{aligned}
B_{2}= & \frac{\eta^{2}}{4}\left(u_{4}-u_{1}-u_{3}+u_{2}-2 u_{6}+2 u_{8}\right) \\
& +\frac{\eta}{2}\left(3 u_{1}+u_{2}-3 u_{3}-u_{4}+4 u_{5}-4 u_{7}\right) \\
& +\frac{1}{2}\left(2 u_{5}+u_{6}+2 u_{7}-u_{1}-u_{2}-u_{3}-u_{4}-u_{8}\right) \\
B_{3}= & \frac{1}{4} A^{2}\left(u_{1}-u_{2}+u_{3}-u_{4}+4 u_{5}-4 u_{7}\right) \\
B_{4}= & \frac{A}{2}\left[\left(3 u_{1}+u_{2}-u_{3}-3 u_{4}-u_{5}+u_{7}\right)\right. \\
& \left.+\eta\left(2 u_{2}+2 u_{3}+2 u_{1}-2 u_{4}+u_{8}-u_{6}\right)\right] \\
B_{5}= & \eta\left(u_{1}+u_{4}-2 u_{8}\right)+\frac{1}{2}\left(u_{4}-u_{1}\right) .
\end{aligned}
$$

Similar expressions for $C_{1}, C_{2}, C_{3}, C_{4}, C_{5}$ can be obtained by replacing $u_{i}$ with $v_{i}$. Substituting eqns (14), (17) into (16) we have

$$
\begin{aligned}
\epsilon_{x}= & \left(B_{1} f_{1}+B_{3} f_{3}\right) r^{2 \lambda-1}+\left(B_{2} f_{1}+B_{4} f_{3}\right) r^{\lambda-1} \\
& +B_{5} f_{3} r^{-1} \\
\epsilon_{y}= & \left(C_{1} f_{2}+C_{3} f_{4}\right) r^{2 \lambda-1}+\left(C_{2} f_{2}+C_{4} f_{4}\right) r^{\lambda-1} \\
& +C_{5} f_{4} r^{-1} \\
\gamma_{x y}= & \left(B_{1} f_{2}+B_{3} f_{4}+C_{1} f_{1}+C_{3} f_{3}\right) r^{2 i-1} \\
& +\left(B_{2} f_{2}+B_{4} f_{4}+C_{2} f_{2}+C_{4} f_{3}\right) r^{\lambda-1} \\
& +\left(B_{5} f_{4}+C_{5} f_{3}\right) r^{-1} .
\end{aligned}
$$

It can be seen that the strains have $r^{-1}, r^{\lambda}-1$ and $r^{2 \lambda-1}$ singularities along any ray. When the constraints $u_{1}=u_{4}=u_{8}$, and $v_{1}=v_{4}=v_{8}$ are imposed strains have $r^{2 \lambda-1}$ and $r^{\lambda-1}$ singularities. The term $r^{2 \lambda-1}$ is nonsingularity for $2 \lambda-1 \geqslant 0$.

\section{CUBIC ISOPARAMETRIC ELEMENT}

Consider now the cubic, 12-node isoparametric element

$$
\begin{aligned}
& x=\sum_{i=1}^{12} N_{i} x_{i}, \quad y=\sum_{i=1}^{12} N_{i} y_{i} \\
& u=\sum_{i=1}^{12} N_{i} u_{i}, \quad v=\sum_{i=1}^{12} N_{i} v_{i},
\end{aligned}
$$

where shape functions are given by [9]. Corner nodes

$$
N_{1}=\frac{1}{32}(1-\xi)(1-\eta)\left[9\left(\xi^{2}+\eta\right)-10\right], \text { etc. }
$$

Mid-side nodes

$$
N_{5}=\frac{9}{32}(1-3 \xi)\left(1-\xi^{2}\right)(1-\eta) \text {, etc. }
$$

When one side of the quadrilateral is collapsed the triangular element of Fig. 2 is formed. We have

$$
\begin{array}{ll}
x_{7}=\frac{2}{3} x_{2}+\frac{1}{3} x_{3}, & y_{7}=\frac{2}{3} y_{2}+\frac{1}{3} y_{3} \\
x_{8}=\frac{1}{3} x_{2}+\frac{2}{3} x_{3}, & y_{8}=\frac{1}{3} y_{2}+\frac{2}{3} y_{3} .
\end{array}
$$

According to $[8]$

$$
\begin{array}{ll}
x_{5}=\alpha x_{2}, & x_{6}=\beta x_{2}, \quad y_{5}=\alpha y_{2}, \quad y_{6}=\beta y_{2} \\
x_{10}=\alpha x_{3}, & x_{9}=\beta x_{3}, \quad y_{10}=\alpha y_{3}, \quad y_{9}=\beta y_{3} .
\end{array}
$$

Substituting eqns (22), (23), (24) into (20) and collecting terms yiclds

$$
\begin{aligned}
x= & \frac{1}{2}\left[x_{2}(1-\eta)+x_{3}(1-\eta)\right]\left[\frac{1}{16}(9 \alpha+9 \beta-1)\right. \\
& +\frac{1}{16}(27 \beta-27 \alpha-1) \xi+\frac{9}{16}(1-\alpha-\beta) \xi^{2} \\
& \left.+\frac{9}{16}(1+3 \alpha-3 \beta) \xi^{3}\right] .
\end{aligned}
$$

The lcast-squares method associated with a simplex method [8] can been used to determine $\alpha$ and $\beta$ values so that

$$
\begin{aligned}
\frac{1}{16}(9 \alpha & +9 \beta-1)+\frac{1}{16}(27 \beta-27 \alpha-1) \xi \\
& +\frac{9}{16}(1-\alpha-\beta) \xi^{2}+\frac{9}{16}(1+3 \alpha-3 \beta) \xi^{3} \\
= & \left(\frac{1+\xi}{2}\right)^{1 / \lambda} .
\end{aligned}
$$

It can seen that eqn (25) is same as eqn (8). Following the procedure outlined before, the strain components can be obtained

$$
\begin{aligned}
\epsilon_{x}= & \left(D_{1} f_{1}+D_{4} f_{3}\right) r^{3 i-1}+\left(D_{2} f_{1}+D_{5} f_{3}\right) r^{2 i-1} \\
& +\left(D_{3} f_{1}+D_{6} f_{3}\right) r^{i-1}+D_{7} f_{3} r^{-1} \\
\epsilon_{y}= & \left(E_{1} f_{2}+E_{4} f_{4}\right) r^{3 \lambda-1}+\left(E_{2} f_{2}+E_{5} f_{4}\right) r^{2 i-1} \\
& +\left(E_{3} f_{2}+E_{6} f_{4}\right) r^{i-1}+E_{7} f_{4} r^{-1}
\end{aligned}
$$

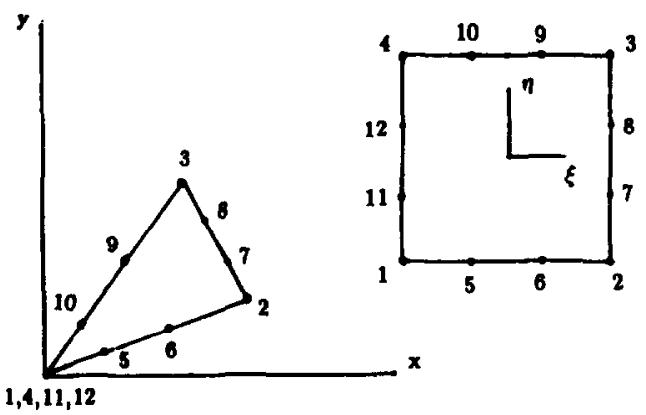

Fig. 2. Collapsed cubic isoparametric element and mapped unit element. 


$$
\begin{aligned}
\gamma_{x y}= & \left(D_{1} f_{2}+D_{4} f_{4}+E_{1} f_{1}+E_{4} f_{3}\right) r^{3 i-1} \\
& +\left(D_{2} f_{2}+D_{5} f_{4}+E_{2} f_{1}+E_{5} f_{3}\right) r^{2 \lambda-1} \\
& +\left(D_{3} f_{2}+D_{6} f_{4}+E_{3} f_{1}+E_{6} f_{3}\right) r^{\dot{\lambda}-1} \\
& +\left(D_{7} f_{4}+E_{7} f_{3}\right) r^{-1}
\end{aligned}
$$

where

$$
\begin{aligned}
& D_{1}=\frac{27}{32} A^{2}\left[(1-\eta)\left(3 u_{5}-3 u_{6}+u_{2}-u_{1}\right)\right. \\
& \left.+(1+\eta)\left(3 u_{10}-3 u_{9}+u_{3}-u_{4}\right)\right] \\
& D_{2}=\frac{18}{27} A\left[(1-\eta)\left(4 u_{1}-2 u_{2}-10 u_{5}+8 u_{6}\right)\right. \\
& \left.+(1-\eta)\left(4 u_{4}-2 u_{3}+8 u_{9}-10 u_{10}\right)\right] \\
& D_{3}=\frac{27}{32}\left[(1-\eta)\left(3 u_{5}-3 u_{6}+u_{2}-u_{1}\right)\right. \\
& \left.+(1+\eta)\left(3 u_{10}-3 u_{9}+u_{4}-u_{3}\right)\right] \\
& -\frac{18}{27}\left[(1-\eta)\left(u_{1}+u_{2}-u_{5}-u_{6}\right)\right. \\
& \left.+(1+\eta)\left(u_{3}+u_{4}-u_{9}-u_{10}\right)\right] \\
& D_{4}=\frac{9}{32} A^{3}\left[u_{1}-u_{2}+u_{3}-u_{4}-3\left(u_{5}-u_{6}+u_{9}-u_{10}\right)\right] \\
& D_{5}=\frac{9}{32} A^{2}\left(2 u_{2}-4 u_{1}+4 u_{4}-2 u_{3}+11 u_{5}\right. \\
& \left.-7 u_{6}+7 u_{9}-11 u_{10}\right) \\
& D_{6}=\frac{A}{32}\left[35\left(u_{1}-u_{4}\right)+u_{2}-u_{3}-72\left(u_{5}-u_{10}\right)\right. \\
& +36\left(u_{6}-u_{9}\right)+27\left(u_{8}-u_{7}-u_{11}-u_{12}\right) \\
& +\left(27 \eta^{2}-18 \eta\right)\left(u_{1}-u_{2}\right)+\left(27 \eta^{2}+18 \eta\right) \\
& \times\left(u_{3}-u_{4}\right)+\left(9 \eta^{2}+2 \eta\right)\left(u_{11}-u_{8}\right) \\
& \left.+\left(9 \eta^{2}-2 \eta\right)\left(u_{7}-u_{12}\right)\right] \\
& D_{7}=\frac{1}{16}\left[\left(1-27 \eta^{2}\right)\left(u_{1}-u_{4}\right)-\left(1-3 \eta^{2}\right)\right. \\
& \left.\times\left(u_{11}-u_{12}\right)+18 \eta\left(u_{1}+u_{4}-u_{11}-u_{12}\right)\right] .
\end{aligned}
$$

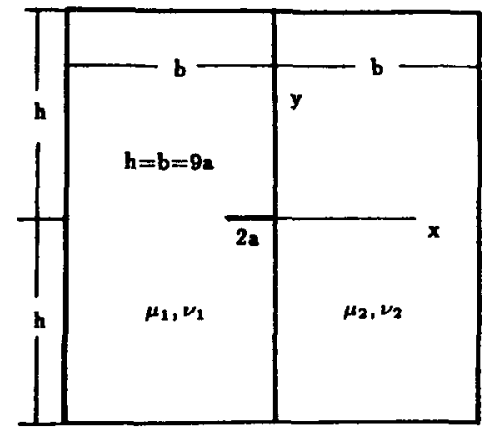

Fig. 3. Cracked bimaterial strip.

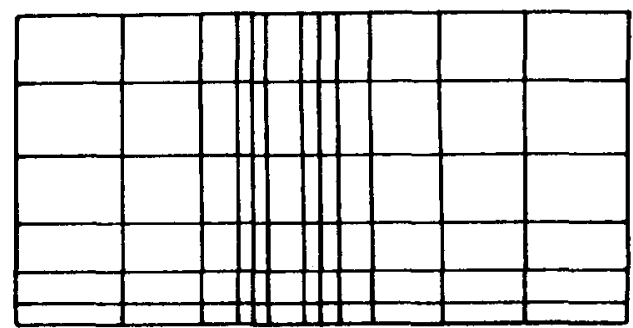

Fig. 4. Finite element mesh.

Similar expressions for $E_{i}$ hold.

It can be seen that strains have $r^{-1}, r^{i-1}, r^{2 i-1}$, $r^{3 \lambda-1}$ singularities along any ray. When the constraints $u_{1}=u_{4}=u_{11}=u_{12}$ and $v_{1}=v_{4}=v_{11}=v_{12}$ are imposed the term $r^{-1}$ is zero. The term $r^{3 i-1}$ is nonsingularity for $3 \lambda-1 \geqslant 0$. Terms $r^{3 i-1}$ and $r^{2 i-1}$ are nonsingularity for $2 \lambda-1 \geqslant 0$.

\section{EXAMPLES}

Consider a bimaterial strip with a crack normal to and terminating at the bimaterial interface as shown in Fig. 3. The finite element mesh is shown in Figs 4 and 5. The total mesh involved 453 nodes and 132 elements. Because of symmetry, it is noted that only the upper half of the strip is analysed. The first numerical example is an epoxy-aluminum combination: $\mu_{1}=0.1667 \times 10^{6} \mathrm{psi}, \quad \mu_{2}=3.846 \times 10^{6} \mathrm{psi}$, $v_{1}=0.35, v_{2}=0.3, m=\mu_{2} / \mu_{1}=23.08, \lambda=0.6619$ and $\alpha=0.35021$ [8]. The crack surfaces are subjected to the uniform pressure. The crack surface displacement is shown in Fig. 6. Our results agree with the results of the singular integral equation [2]. The stress intensity factor generalized for both the embedded and bondline crack tips is defined as

$$
K=\sqrt{2 \pi} \lim _{r \rightarrow 0} r^{1-i} \sigma_{y y}(r, 0) .
$$

To reduce $K$ from the displacement data the following equation was used

$$
K=\sqrt{2 \pi} \mu * u_{y}(r, \pi) / r^{\dot{2}},
$$

where

$$
\mu^{*}=\mu_{2} \frac{(1-2 \lambda)\left(m+k_{2}\right)+(1+2 \lambda)\left(1+m k_{1}\right)}{\left(m+k_{2}\right)\left(1+m k_{1}\right) \sin \pi \hat{\lambda}} .
$$

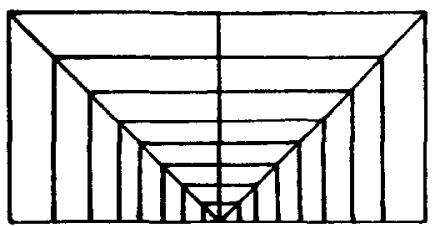

Fig. 5. Mesh in crack location. 


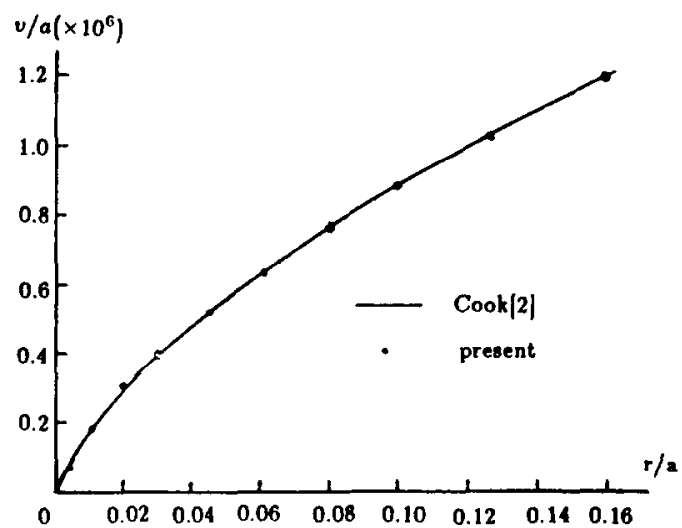

Fig. 6. Crack surface displacement.

From the equation the normalized stress intensity factor $K / \sigma \sqrt{\pi} a^{1-\lambda}$ at the bondline crack ends computed from the finite element data at $r / a=0.01$ was found to equal 2.752. The SIE displacement data [2] and special triangular element [4] predict 2.7845 and 2.85 , respectively. It is 2.708 by using Abdi's quadratical element [8]. The second example is aluminum-steel combination. In that case we have $\mu_{2}=3 \mu_{1}, v_{2}=0.3, \lambda=0.5993, \alpha=0.314$. Uniform tension $\sigma_{y 1}$ and $\sigma_{y 2}$ are applied the ends. To assure the continuity of deformation at the end, $\sigma_{y 2}$ is taken as $\sigma_{y 1} E_{2} / E_{1}$ for plane strain assumption. The value of the normalized intensity factor $K / \sigma \sqrt{\pi} a^{1-\lambda}$ was found to be 1.930 which agree well with 1.938 computed by the hybrid finite element model [11]. The Abdi's element gives 1.896 .

\section{CONCLUSION}

It has been demonstrated that the variable power singularity of a strain field at the crack tip can be obtained simply. Numerical examples show that the present approach makes possible a highly accurate and efficient numerical solution of variable power singular problems.

Acknowledgments - This work was supported by the National Natural Science Foundation of China and Laboratory for Nonlinear Mechanics of Continuous Media, Institute of Mechanics, Chinese Academy of Sciences.

\section{REFERENCES}

I. A. R. Zak and M. L. Williams, Crack point stress singularities at a bi-material interface. J. Appl. Mech. 30, 142-143 (1963).

2. T. S. Cook and F. Erdogan, Stress in bonded materials with crack perpendicular to the interface. Int. J. Engng Sci. 10, 677-697 (1972).

3. J. E. Akin, The generation of elements with singularities. Int. J. Numer. Meth. Engng 10, 1249-1259 (1976).

4. D. M. Tracey and T. S. Cook, Analysis of power type singularities using finite elements. Int. J. Numer. Meth. Engng 11, 1125-1233 (1977).

5. M. Stern, Families of consistent elements with singular derivative fields. Int. J. Numer. Meth. Engng 14, 409-422 (1979).

6. T. J. Hughes and J. E. Akin, Techniques for developing 'special' finite element shape functions with particular reference to singularities. Int. J. Numer. Meth. Engng 15 (1980).

7. G. H. Staab, A variable power singular element for analysis of fracture mechanics problems. Comput. Struct. 17, 449-457 (1983).

8. R. El Abdi and G. Valentin, Isoparametric elements for crack normal to the interface between two bonded layers. Comput. Struct. 33, 241-248 (1989).

9. O. C. Zienkiewicz, The Finite Element Method, 3rd Edn. McGraw-Hill. New York (1977).

10. Y. Yamada, Y. Ezawa, I. Nishigachi and M. Okabe, Reconsiderations on singularity or crack tip elements. Int. J. Numer. Meth. Engng 14, 1520-1544 (1979).

11. Wen-hwa Chen, Kuen-tsann Chen and Chun-ron Chang, On the applicability of integral for perpendicular interface crack. Engng Fracture Mech. 30, 13-19 (1988). 